\title{
Association of Elite Sports Status with Gene Variants of Peroxisome Proliferator Activated Receptors and Their Transcriptional Coactivator
}

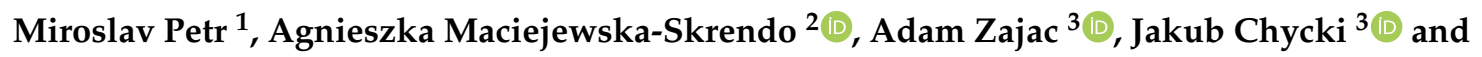 \\ Petr Stastny ${ }^{1, *(D)}$ \\ 1 Faculty of Physical Education and Sport, Charles University, 16252 Prague, Czech Republic; \\ petr@ftvs.cuni.cz \\ 2 Faulty of Physical Education, Gdansk University of Physical Education and Sport, 80-336 Gdansk, Poland; \\ maciejewska.us@wp.pl \\ 3 Department of Theory and Practice of Sport, The Jerzy Kukuczka Academy of Physical Education, \\ 40-065 Katowice, Poland; a.zajac@awf.katowice.pl (A.Z.); j.chycki@awf.katowice.pl (J.C.) \\ * Correspondence: stastny@ftvs.cuni.cz; Tel.: +420-777-198-764
}

Received: 21 October 2019; Accepted: 21 December 2019; Published: 25 December 2019

\begin{abstract}
Background: Although the scientific literature regarding sports genomics has grown during the last decade, some genes, such as peroxisome proliferator activated receptors (PPARs), have not been fully described in terms of their role in achieving extraordinary sports performance. Therefore, the purpose of this systematic review was to determine which elite sports performance constraints are positively influenced by PPARs and their coactivators. Methods: The Preferred Reporting Items for Systematic Reviews and Meta-Analyses guidelines were used, with a combination of PPAR and sports keywords. Results: In total, 27 studies that referred to PPARs in elite athletes were included, where the Ala allele in PPARG rs1801282 was associated with strength and power elite athlete status in comparison to subelite athlete status. The $\mathrm{C}$ allele in PPARA rs4253778 was associated with soccer, and the $\mathrm{G}$ allele PPARA rs4253778 was associated with endurance elite athlete status. Other elite status endurance alleles were the Gly allele in PPARGC1A rs8192678 and the C allele PPARD rs2016520. Conclusions: PPARs can be used for estimating the potential to achieve elite status in human physical performance in strength and power, team, and aerobic sports disciplines. Carrying specific PPAR alleles can provide a partial benefit to achieving elite sports status, but does not preclude achieving elite status if they are absent.
\end{abstract}

Keywords: PPAR; human performance; aerobic training; genetic predisposition; muscle fibers; anaerobic training; power; endurance training; adaptation; strength training

\section{Introduction}

The scientific literature on exercise genomics has shown clear evidence that genetic markers are associated with endurance [1], power athlete status [2,3], trainability [4], and even psychological factors [5], and peroxisome proliferator activated receptors (PPARs) and/or their coactivators are often listed. While both sports performance and genomics are highly multifactorial domains, it is beneficial to summarize what phenotypic domains can be attributed to PPARs (and their coactivators) and where the analysis of phenotypic domains is redundant. Moreover, better knowledge, via functional genomics, of how PPARs (and their coactivators) may affect the individual response to physical activity or environmental factors is highly relevant not only for active individuals (athletes), but also for people who are undergoing a health treatment program that includes a physical intervention [6]. In this context, data from athletes can serve as a basis for hypotheses regarding the effectiveness of physical 
activity programs under extreme physiological conditions or for sedentary individuals, where the clear objective is to achieve health improvement. A recent review on the role of PPAR polymorphisms in trainability summarized several studies showing genotype/allele specific changes in health related markers [4].

PPARs are a subfamily of nuclear hormone receptors that form heterodimers with retinoid $X$ receptors and regulate the transcription of several genes involved in lipid metabolism, energy utilization, and storage [7]. PPARs also regulate genes for glucose metabolism, carcinogenesis, and inflammation $[8,9]$. There are three isoforms of PPARs (PPAR $\alpha$, PPAR $\beta / \delta$, and PPAR $\gamma$, encoded by the PPARA, PPARD, and PPARG genes, respectively) that differ in their distribution and function [10]. For example, PPAR $\gamma$ is predominantly active in fat cells where it affects differentiation and growth; among other things, it is also an interesting target in pharmacotherapy for diabetes mellitus type 2 (DM2) [11]. An increased level of PPAR expression occurs in tissues that catabolize high amounts of fatty acids, such as the liver, kidney, brown adipose tissue, heart, and skeletal muscle [12,13]. In addition, muscle specific PPAR $\beta / \delta$ overexpression is considered to be a part of skeletal muscle plasticity. Therefore, the role of PPARs in elite aerobic performance is highly suspected [1,14].

The peroxisome proliferator activated receptor $\gamma$ coactivator 1 (PGC1) family of transcriptional coactivators, consisting of three members, PGC1 $\alpha$, PGC1 $\beta$, and the PGC- 1 related coactivator (PRC), encoded by the PPARGC1A, PPARGC1B, and PPRC1 genes, respectively, provides important links between these transcription factors and the physiological signals controlling cellular functions related to cellular and mitochondrial energy metabolism $[15,16]$. PGC1 $\alpha$ is the most frequently studied and positively regulates mitochondrial biogenesis and respiration and many other metabolic processes, including adaptive thermogenesis, gluconeogenesis, and insulin signaling [17].

The links between PPARs (and their coactivators) and muscle morphology [18], oxygen uptake [19,20], power output [21], endurance performance [18], and human trainability [4] have already been associated with elite sports status in individual studies and, in the case of PPARGC1A Gly428Ser, by systematic review with meta-analyses [22]. Therefore, there has been an increase of PPAR analyses in the athletic population in recent years, where PPARA, PPARG, PPARD, and their transcriptional coactivators' PPARGC1A and PPARGC1B gene polymorphisms contribute to the observed phenotypes. For example, it has been shown that prolonged endurance exercise increases the transcriptional activity of PPARGC1A in active subjects [23]. In contrast, a recent systematic review on genes related to the level of endurance performance in mice considered at least three PPAR gene variants (and their coactivators) to be associated with endurance capacity [24]. Previous literature reviews [2,3] focused on all possible genes that might have an association with strength and power athletes' status and suggested that PPARs have important roles that require detailed analyses. So far, only PPARGC1A has been reviewed in relation to power athlete status [22], and endurance athlete status and other PPARs and their coactivators have not.

Since the scientific literature in sports genomics has grown during the last decade, some genes, such as PPARs and/or their coactivators, have not been adequately described in terms of their role in athlete training and achieving extraordinary sports performance. Therefore, the purpose of this systematic review was to determine which PPARs and their coactivators are positively or negatively associated with elite sports performance constraints. We hypothesize that PPARs and/or their coactivators might determine aerobic performance and team sports elite athlete status, but not speed and strength oriented elite athlete status.

\section{Results}

The literature search resulted in a total of 4916 articles, after removing duplicates. The number of eligible articles was further reduced to 79 (including 31 reviews) after screening article titles and abstracts according to the inclusion criteria that the articles include PPARs and/or their coactivators' gene polymorphisms at the elite athlete level (Figure 1). Of these studies, 18 were rejected following the full-text screening, and three were rejected based on the methodological quality criteria. Finally, 27 studies (Figure 1) were included in the analysis. 


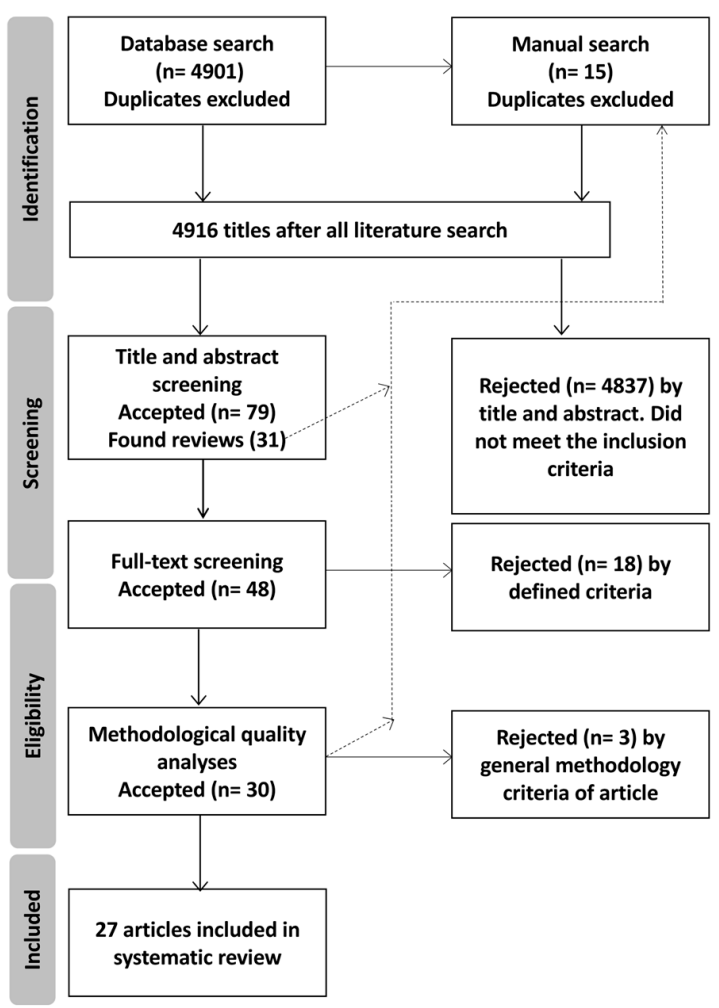

Figure 1. Flowchart of the review for the articles included in the tables. The dotted line demonstrates the stages where a manual search of the reference lists of the selected articles was performed.

In total, 27 studies were included due to referring to PPARs with elite athlete status, where five PPARs were summarized as the main result of qualitative synthesis (Table 1). Thus, PPARs and their coactivators determined aerobic, speed, strength, and team sports elite athlete status. In total, 11 studies found differences between elite and subelite athletes or among elite athletes from different disciplines (Table 2). The comparison between PPARs in elite athletes and control groups only was reported in 14 studies (Table 3) and supported the main conclusions of this study. One study was a single case study and one without a control group (Table 3). The Ala allele in PPARG rs1801282 and the $C$ allele in PPARA rs4253778 were associated with strength and power elite athlete status in comparison to subelite athletes' status (Tables 1 and 2). The G allele PPARA rs4253778, Gly allele in PPARGC1A rs8192678, and the PPARD rs2016520 C allele were associated with endurance elite athlete status in comparison to subelite athlete status (Tables 1 and 2). The $C$ allele in PPARA rs4253778 was associated with mixed endurance/strength-power (soccer) such as the PPARD A/C/C haplotype in rs2016520, rs2267668, and rs1053049, however only in comparison to control groups (Tables 1-3). In contrast, the $\mathrm{G}$ allele in PPARA rs4253778 was associated with mixed endurance/strength-power (soccer) with other elite athletes from combat sports and motorcycling. 
Table 1. Alleles and genotypes related to elite athlete status vs. subelite status in different types of disciplines and in comparison to controls. ${ }^{*}$ The minority report results specific for the reported population.

\begin{tabular}{cll}
\hline & Elite Athlete vs. Subelite Athlete & Elite Athlete vs. Controls \\
\hline & & $P P A R G$ rs1801282 Ala allele \\
Strength and power oriented & $P P A R G$ rs1801282 Ala allele & $P P A R A 7$ rs4253778 C allele \\
& $P P A R A$ rs4253778 C allele & $P P A R A$ rs4253778* GG genotype, G allele \\
& & $P P A R A$ rs4253778 C allele \\
& & $P P A R G C 1 A$ rs8192678 Gly/Gly genotype \\
\hline \multirow{3}{*}{ Endurance oriented } & $P P A R A$ rs4253778 G allele & $P P A R$ rs4253778* C allele \\
& $P P A R D$ rs2016520 C allele & $P P A R D$ rs2016520 C allele \\
& $P P A R G C 1 A$ rs8192678 Gly allele, Gly/Gly genotype & $P P A R G C 1 B$ rs773267 C allele \\
& & $P P A R A$ rs4253778 GG genotype, G allele \\
Mixed endurance/power & & $P P A R G C 1 A$ rs8192678 Gly4 allele, Gly/Gly genotype \\
& & $P P A R A$ rs4253778 C allele, CC genotype \\
& & $P P A R D$ haplotypes: \\
& & rs2016520, rs2267668, rs1053049 A/C/C \\
\hline
\end{tabular}

Table 2. PPAR alleles and genotypes in elite and subelite athletes and their differences among disciplines. TGS, total genetic score.

\begin{tabular}{|c|c|c|c|}
\hline Participant Type $(n)$ & Gene/Variation & Results & Authors \\
\hline $\begin{array}{l}\text { Russian endurance (swimming, track-and-field, } \\
\text { triathlon, cross-country skiing, biathlon, skating, } \\
\text { road cycling (390), and strength oriented athletes } \\
\text { (rowing, boxing, ice-hockey, wrestling, court } \\
\text { tennis, weightlifting (396); controls: (1242) }\end{array}$ & $P P A R A$ rs4253778 (intron 7G/C) & $\begin{array}{l}\text { C allele: endurance oriented }<\text { controls }(p<0.0001) \\
\text { C allele: power oriented }>\text { controls }(p<0.0001) \\
\text { CC genotype: mixed endurance/power oriented }>\text { controls }(p= \\
0.0012) \\
\text { C allele: increasing with anaerobic component }(p<0.029) \\
\text { C allele: increasing frequency in power oriented elite athletes } \\
(p=0.0316) \\
\text { G allele: increasing frequency in endurance oriented elite athletes } \\
(p<0.0001)\end{array}$ & $\begin{array}{l}\text { Ahmetov et al., } \\
2006[25]\end{array}$ \\
\hline $\begin{array}{l}\text { Russian elite, subelite athletes, and nonelite } \\
\text { athletes (1539); controls (610) }\end{array}$ & $\begin{array}{l}\text { PPARD rs2016520 } \\
(+294 \mathrm{~T} / \mathrm{C})\end{array}$ & $\begin{array}{l}\text { C allele: athletes }>\text { controls }(p<0.0001) \\
\text { C allele: athletes in endurance oriented sports }>\text { controls } \\
(p<0.0001) \\
\text { C allele: cyclic endurance oriented elite sports }>\text { subelite }(p<0.01) \\
C \text { allele: most pronounced between high and top level } \\
\text { long-distance athletes }(p=0.013)\end{array}$ & $\begin{array}{l}\text { Ahmetov et al., } \\
2007 \text { [26] }\end{array}$ \\
\hline
\end{tabular}


Table 2. Cont.

\begin{tabular}{|c|c|c|c|}
\hline Participant Type ( $n$ ) & Gene/Variation & Results & Authors \\
\hline $\begin{array}{l}\text { Russian athletes of various strength and speed } \\
\text { disciplines (260); } \\
\text { controls (1073) }\end{array}$ & $\begin{array}{l}\text { PPARG rs1801282 } \\
\text { (Pro12Ala) }\end{array}$ & $\begin{array}{l}\text { 12Ala allele: athletes }>\text { controls }(p<0.0001) \\
\text { 12Ala allele: skate sprinters }(p=0.0002) \text {, throwers }(p=0.012) \text {, } \\
\text { weightlifters }(p=0.003)>\text { controls } \\
\text { 12Ala allele: honored masters of sports }>\text { masters of sports of } \\
\text { international rank }>\text { masters of sports }>\text { candidate masters of } \\
\text { sports }(p<0.0001)\end{array}$ & $\begin{array}{l}\text { Ahmetov et al., } \\
2008 \text { [27] }\end{array}$ \\
\hline $\begin{array}{l}\text { Russian long endurance (cycling, biathlon, } \\
\text { triathlon, long distance racing) and middle } \\
\text { endurance ( } 3-10 \text { km runners, skaters, } \\
5-10 \text { km cross-country skiers, } 800-1500 \mathrm{~m} \\
\text { swimmers) athletes (577); } \\
\text { controls (1132) }\end{array}$ & $\begin{array}{l}\text { PPARA rs4253778 (intron 7G/C) } \\
\text { PPARD rs2016520 (+294T/C) } \\
\text { PPARG rs1801282 (missense C/G) } \\
\text { PPARGC1A rs8192678 (missense A/G) } \\
\text { PPARGC1B rs7732671 (missense C/G) } \\
\text { interactions of } 10 \text { genetic } \\
\text { polymorphisms }\end{array}$ & $\begin{array}{l}\text { C allele: long endurance athletes }<\text { non-athletes }(p=0.018) \\
\text { C allele: long endurance athletes }>\text { h }(p=0.006) \\
\text { NS } \\
\text { A (Ser) allele: long endurance athletes }<\text { non-athletes }(p<0.001) \\
\text { C allele: long endurance athletes }>\text { non-athletes }(p=0.004) \\
\text { High number }(\geq 9) \text { of "endurance" alleles: long endurance elite }> \\
\text { subelite }>\text { nonelite }(p=0.01) \\
\text { High number }(\geq 9) \text { of "endurance" alleles: middle endurance elite } \\
>\text { subelite }>\text { nonelite }(p=0.003)\end{array}$ & $\begin{array}{l}\text { Ahmetov et al., } \\
2009 \text { [18] }\end{array}$ \\
\hline $\begin{array}{l}\text { Polish elite and subelite combat athletes (60); } \\
\text { controls (181) }\end{array}$ & $\begin{array}{l}\text { PPARA rs } 4253778 \\
\text { (intron } 7 \mathrm{G} / \mathrm{C} \text { ) }\end{array}$ & $\begin{array}{l}\text { GG genotype: athletes }>\text { controls }(p=0.04) \\
\text { G allele: athletes }>\text { controls }(p=0.01)\end{array}$ & $\begin{array}{l}\text { Cieszczyk et al., } \\
2011[28]\end{array}$ \\
\hline $\begin{array}{l}\text { Italian elite athletes (combat sports, motorcycle, } \\
\text { soccer) (113); controls not included }\end{array}$ & $\begin{array}{l}\text { PPARA (rs4253778) } \\
\text { (intron } 7 \mathrm{G} / \mathrm{C})\end{array}$ & $\begin{array}{l}\text { GG genotype: soccer }>\text { combat sports and motorcycle } \\
\text { G allele: soccer }>\text { combat sports and motorcycle }\end{array}$ & $\begin{array}{l}\text { Cocci et al., } 2019 \\
\text { [29] }\end{array}$ \\
\hline $\begin{array}{l}\text { Ukrainian elite, subelite athletes, and nonelite, } \\
\text { endurance and power oriented athletes (210); } \\
\text { controls (326) }\end{array}$ & $\begin{array}{l}\text { PPARA rs4253778 (intron 7G/C) } \\
\text { PPARG rs1801282 } \\
\text { (Pro12Ala) } \\
\text { PPARGC1B rs7732671 (Ala2032Pro) } \\
\text { Total genetic score of } 6 \text { gene } \\
\text { polymorphisms }\end{array}$ & $\begin{array}{l}\text { NS } \\
\text { 12Ala allele: power oriented }>\text { endurance oriented }(p=0.008) \\
\text { NS } \\
\text { TGS: power oriented athletes }>\text { control }(p=0.0142)\end{array}$ & $\begin{array}{l}\text { Drozdovska et } \\
\text { al., } 2013[30]\end{array}$ \\
\hline $\begin{array}{l}\text { Israeli national/international track-and-field } \\
\text { athletes (155); controls } 240\end{array}$ & $\begin{array}{l}\text { PPARA rs4253778 (intron 7G/C) } \\
\text { PPARGC1A rs8192678 (Gly482Ser) }\end{array}$ & $\begin{array}{l}\text { Associated with endurance performance CI } 95 \% \\
\text { Gly allele: endurance }>\text { controls }(p<0.05 *) \\
\text { Gly/Gly genotype: endurance }>\text { strength oriented }>\text { controls } \\
(p<0.05 *) \\
\text { Associated with endurance performance CI } 95 \% . \\
\text { NS } \\
\text { TGS: endurance athletes }>\text { control and power athletes }(p<0.001) \\
\text { elite status NS }\end{array}$ & $\begin{array}{l}\text { Eynon et al., } \\
2011[31]\end{array}$ \\
\hline
\end{tabular}


Table 2. Cont

\begin{tabular}{|c|c|c|c|}
\hline Participant Type ( $n$ ) & Gene/Variation & Results & Authors \\
\hline $\begin{array}{l}\text { Israeli track-and-field athletes (155); controls } \\
\text { (240) }\end{array}$ & $\begin{array}{l}\text { PPARA rs4253778 (intron 7G/C) } \\
\text { PPARGC1A rs8192678 (Gly482Ser) }\end{array}$ & $\begin{array}{l}\text { NS } \\
\text { Ser/Ser genotype: endurance athletes }<\text { sprinters }(p=0.016) \text { and } \\
\text { controls }(p=0.012) \\
\text { Gly allele, Gly/Gly genotype elite athletes }>\text { non elite }(p=0.02)\end{array}$ & $\begin{array}{l}\text { Eynon et al., } \\
2010 \text { [32] }\end{array}$ \\
\hline $\begin{array}{l}\text { Russian elite, subelite, and nonelite soccer } \\
\text { players (246); controls ( } 872)\end{array}$ & $\begin{array}{l}\text { PPARD rs2016520 (T294C) } \\
\text { PPARG rs1801282 (Pro12Ala) } \\
\text { PPARGC1A rs8192678 (Gly482Ser) } \\
\text { Total genetic score of } 8 \text { gene } \\
\text { polymorphisms } \\
\text { Gene/Variation }\end{array}$ & $\begin{array}{l}\text { CC genotype: soccer players }>\text { controls }(p=0.0001) \\
\text { C allele: soccer players }>\text { controls }(p=0.0007) \\
\text { C allele: attackers }>\text { controls }(p<0.0001) \\
\text { C allele: elite soccer players }>\text { controls }(p=0.007) \\
\text { NS } \\
\text { NS } \\
\text { NS } \\
\text { TGS: elite soccer players }>\text { subelite }>\text { nonelite }(p=0.002) \\
\text { TGS: elite soccer goalkeepers and midfielders }>\text { subelite }> \\
\text { nonelite }(p=0.002) \\
\text { Results }\end{array}$ & $\begin{array}{l}\text { Egorova et al., } \\
2013 \text { [33] }\end{array}$ \\
\hline $\begin{array}{l}\text { Lithuanian athletes, endurance (biathlon, } \\
\text { pentathlon, road cycling, cross-country skiing, } \\
\text { swimming, rowing, track-and-field long } \\
\text { distance) } \\
\text { power (weightlifting, track-and-field short } \\
\text { distance) } \\
\text { mixed (tennis, handball, boxing, wrestling, } \\
\text { football) (193); controls (250) }\end{array}$ & $\begin{array}{l}\text { PPARGC1A } \\
\text { rs8192678 (Gly482Ser) } \\
\text { PPARA rs4253778 (intron 7G/C) }\end{array}$ & $\begin{array}{l}\text { Gly/Gly }<\text { Ser/Ser genotypes: anaerobic alactic maximum power } \\
\text { (AAMP) in endurance and power athletes }(p=0.024) \\
\text { C allele: athletes }>\text { controls }(p=0.046) \\
\text { CC genotype: nonelite }<\text { subelite }<\text { elite }\end{array}$ & $\begin{array}{l}\text { Gineviciene et } \\
\text { al., } 2011 \text { [34] }\end{array}$ \\
\hline
\end{tabular}

The * meaning is: only minimal required significance reported. 
Table 3. Results for PPAR alleles and genotypes in elite athletes and controls.

\begin{tabular}{|c|c|c|c|}
\hline Participant Type $(n)$ & Gene/Variation & Results & Authors \\
\hline $\begin{array}{l}\text { Israeli track-and-field athletes (155); controls } \\
(240)\end{array}$ & PPARA rs135539 (intron $1 \mathrm{~A} / \mathrm{C}$ ) & NS & Eynon et al., 2011 [35] \\
\hline $\begin{array}{l}\text { Lithuanian professional male footballers } \\
\text { (199); controls (167) }\end{array}$ & $\begin{array}{l}\text { PPARGC1A } \\
\text { rs8192678 (Gly482Ser) } \\
\text { PPARA rs4253778 (intron 7C/G) }\end{array}$ & $\begin{array}{l}\text { Gly/Gly genotype: forwards }>\text { controls }(p=0.044) \\
\text { GG genotype: controls }>\text { forwards }(p=0.034)\end{array}$ & Gineviciene et al., 2014 [36] \\
\hline $\begin{array}{l}\text { Russian powerlifters, weightlifters, throwers } \\
\text { (161); controls (1202) }\end{array}$ & $\begin{array}{l}\text { PPARGC1A } \\
\text { rs8192678 (Gly482Ser) }\end{array}$ & $\begin{array}{l}\text { Gly/Gly genotype: powerlifters }>\text { controls }(p=0.002) \\
\text { Weightlifters and throwers no difference from controls }\end{array}$ & Gineviciene et al., 2016 [37] \\
\hline $\begin{array}{l}\text { African and Spanish cross-country runners } \\
\text { of different levels, one world champion (9) } \\
\text { (case study) }\end{array}$ & $\begin{array}{l}\text { PPARGC1A } \\
\text { rs8192678 (Gly482Ser) }\end{array}$ & $\begin{array}{l}\text { Gly/Gly genotype: present in the world champion, but } \\
\text { not in all of the top cross-country runners }\end{array}$ & Gonzales Freire et al. [38] \\
\hline $\begin{array}{l}\text { Mixed nation elite endurance triathletes } \\
\text { (196); controls not included }\end{array}$ & $\begin{array}{l}\text { PPARGC1A } \\
\text { rs8192678 (Gly482Ser) } \\
\text { Total Genetic Score of } 7 \text { gene } \\
\text { polymorphisms }\end{array}$ & $\begin{array}{l}\text { NS } \\
\text { TGS was not significantly associated with performance } \\
\text { time }\end{array}$ & Grealy et al., 2015 [39] \\
\hline $\begin{array}{l}\text { Spanish male endurance athletes (104); } \\
\text { controls (200) }\end{array}$ & $\begin{array}{l}\text { PPARGC1A } \\
\text { rs8192678 (Gly482Ser) }\end{array}$ & Ser482 allele: athletes $<$ unfit controls $(p=0.01)$ & Lucia et al., 2015 [40] \\
\hline Polish rowers (55); controls (115) & PPARA rs4253778 (intron 7C/G) & $\begin{array}{l}\text { GG genotype: elite rowers }>\text { controls }(p=0.04) \\
\text { G allele: all rowers }>\text { controls }(p=0.03) \\
\text { G allele: elite rowers }>\text { controls }(p=0.01)\end{array}$ & Maciejewska et al., 2011 [41] \\
\hline $\begin{array}{l}\text { Polish and Russian athletes of various } \\
\text { disciplines (1605); controls (1816) }\end{array}$ & $\begin{array}{l}\text { PPARGC1A } \\
\text { rs8192678 (Gly482Ser) }\end{array}$ & Ser482 allele: athletes $<$ unfit controls $(p=0.0001)$ & Maciejewska et al., 2012 [42] \\
\hline $\begin{array}{l}\text { Polish athletes (endurance, } \\
\text { strength-endurance, speed-power, } \\
\text { sprint-strength, strength, 660); controls (684) }\end{array}$ & $\begin{array}{l}\text { PPARG rs1801282 } \\
\text { (Pro12Ala) }\end{array}$ & 12Ala allele: strength athletes $>$ controls $(p=0.0007)$ & Maciejewska et al., 2013 [43] \\
\hline $\begin{array}{l}\text { Polish athletes (endurance, } \\
\text { strength-endurance, speed-power, sprinters, } \\
\text { 660); controls (704) }\end{array}$ & $\begin{array}{l}\text { PPARD rs2016529 } \\
\text { PPARD rs1053049 } \\
\text { PPARD rs } 2267668 \\
\text { haplotypes rs2267668/ } \\
\text { rs2016520/rs } 1053049\end{array}$ & $\begin{array}{l}\text { rs2016529 CC genotype: athletes }>\text { controls }(p<0.00001) \\
\text { rs1053049 TT genotype: athletes }>\text { controls }(p<0.0001) \\
\text { NSFG } \\
\text { haplotype A/C/C: athletes }<\text { controls }(p<0.000001)\end{array}$ & Maciejewska et al., 2014 [44] \\
\hline
\end{tabular}


Table 3. Cont

\begin{tabular}{|c|c|c|c|}
\hline Participant Type ( $n$ ) & Gene/Variation & Results & Authors \\
\hline $\begin{array}{l}\text { Spanish professional cyclists, Olympic-class } \\
\text { runners, world-class rowers (141); controls } \\
\text { (123) }\end{array}$ & $\begin{array}{l}\text { PPARGC1A } \\
\text { rs8192678 (Gly482Ser) }\end{array}$ & NS & Muniesa et al., 2010 [45] \\
\hline $\begin{array}{l}\text { Polish elite athletes of different sports } \\
\text { disciplines: power and endurance (413); } \\
\text { controls (451) }\end{array}$ & $\begin{array}{l}\text { PPARGC1A } \\
\text { rs8192678 (Gly482Ser) } \\
\text { PPARG rs1801282 } \\
\text { (Pro12Ala) }\end{array}$ & $\begin{array}{l}\text { NS } \\
\text { NS }\end{array}$ & Peplonska et al., 2017 [46] \\
\hline $\begin{array}{l}\text { Spanish world-class rowers (39); controls } \\
\text { (123) }\end{array}$ & $\begin{array}{l}\text { PPARGC1A } \\
\text { rs8192678 (Gly482Ser) }\end{array}$ & NS & Santiago et al., 2010 [47] \\
\hline $\begin{array}{l}\text { Greek endurance athletes (438); controls not } \\
\text { included }\end{array}$ & $\begin{array}{l}\text { PPARGC1A } \\
\text { rs8192678 (Gly482Ser) } \\
\text { PPARA rs } 4253778 \text { (intron 7C/G) } \\
\text { PPARD rs2267668 } \\
\text { PPARD rs6902123 } \\
\text { PPARD rs1053049 }\end{array}$ & $\begin{array}{l}\text { NS } \\
\text { NS } \\
\text { NS } \\
\text { NS } \\
\text { NS }\end{array}$ & Tsianos et al., 2010 [48] \\
\hline $\begin{array}{l}\text { Turkish elite level endurance athletes (60); } \\
\text { controls (110) }\end{array}$ & $\begin{array}{l}\text { PPARA rs4253778 (intron 7C/G) } \\
\text { PPARGC1A } \\
\text { rs8192678 (Gly482Ser) }\end{array}$ & $\begin{array}{l}\text { GG genotype: athletes }>\text { controls }(p=0.006) \\
\text { G allele: athletes }>\text { controls }(p<0.001) \\
\text { Gly/Gly genotype: athletes }<\text { controls }(p<0.001) \\
\text { Gly482 allele: athletes }<\text { controls }(p<0.001)\end{array}$ & Tural et al., 2014 [49] \\
\hline $\begin{array}{l}\text { Japanese endurance track-and-field athletes } \\
\text { (175); controls (645) }\end{array}$ & $\begin{array}{l}\text { PPARD rs2016520 } \\
(+294 \mathrm{~T} / \mathrm{C}) \\
\text { PPARGC1A } \\
\text { rs8192678 (Gly482Ser) } \\
\text { PPARGC1B rs7732671 } \\
\text { (Ala2032Pro) } \\
\text { Total Genetic Score of } 20 \text { gene } \\
\text { polymorphisms }\end{array}$ & $\begin{array}{l}\text { NS } \\
\text { NS } \\
\text { NS } \\
\text { NS }\end{array}$ & Yvert et al., 2016 [50] \\
\hline
\end{tabular}




\section{Discussion}

The main finding of this review was that PPARs and their coactivator gene polymorphisms were related to the ability to achieve elite sports status for endurance, strength, power, and team sports oriented athletes. This consideration was specifically important for the $\mathrm{C}$ allele in PPARA rs4253778, the $\mathrm{G}$ allele PPARA rs4253778, the Gly allele in PPARGC1A rs8192678, and the C allele PPARD rs2016520, as those alleles have been found in higher frequencies in elite athletes than in subelite athletes (not just controls) and in studies including a large number of PPARs in the optimal genotype score $[30,31,33]$ or haplotype [44]. Other findings (Tables 1 and 3), where the genotype frequency differed between elite athletes and controls, were questionable; however, they still supported the hypothesis that PPAR alleles could influence extreme physical fitness phenotypes. Such an example was given by three studies devoted to the PPARGC1B gene in which two of them showed no association with athletes' status [30,50]. However, the study of Ahmetov [18] examined the total genotype score of 15 genetic variants, where the PPARGC1B C allele was shown to be more common in a group of long endurance athletes compared to sedentary controls.

Although this study identified four alleles that were beneficial for elite athletes, missing the allele or the dominance of endurance or power genotypes does not mean that an athlete cannot achieve elite status, e.g., Eynon [51] reported a case study showing that athletes with the ACTN3 R577X heterozygote variation and five out of six "endurance oriented" genotypes (including PPARs) could be successful in a long 10,000 m and short $400 \mathrm{~m}$ run; similarly, elite long jumpers without the power associated ACTN3 genotype X577X have been reported [52]. On the other hand, Gonzales reported the presence of the Gly/Gly PPARGC1A rs8192678 genotype in a world-champion cross-country runner [38], but admitted that this genotype was not present in other elite runners. Therefore, our results can identify the potential to achieve elite sports levels, since those genotypes are also related to training response [4], but cannot play a role in whole talent identification.

The presence of the Ala allele PPARG rs1801282 and the C allele PPARA rs4253778 in elite athletes might be related to the molecular mechanisms required to sustain high anaerobic training loads [53]. Although PPARG rs1801282 Ala allele carriers have been found in individuals with better reactions to aerobic training in the typical population [54-57], their association in elite athletes might be related to the sustainability of periodic training, which requires tissue recovery and frequent training. Anaerobic training is accompanied by an increase in inflammatory markers, which are regulated by PPARs [8,9]. One study reported the association of the $\mathrm{G}$ allele in PPARA rs4253778 with power oriented sports (combat sports) in a comparison between elite athletes and controls (Table 2) [28], which might be explained by the mixed requirements of this sports discipline. However, this increased frequency has not been reported between elite and subelite athletes.

Most documented genetic predispositions to elite performance have been found in endurance athletes, where the $\mathrm{G}$ allele of PPARA rs4253778, the $\mathrm{C}$ allele of PPARD rs2016520, the Gly allele, and the Gly/Gly genotype of PPARGC1A rs8192678 have been associated with this status as candidate genes and as the crucial part of total genetic scores $[30,31,33]$. This confirmed the observations that the rs 8192678 Gly allele may be a key element associated with the efficiency of aerobic metabolism; however, the question of how the rs8192678 Gly and Ser variants affect cardiorespiratory capacity remains unknown, although engagement of the PGC- $1 \alpha$ coactivator in the regulation of energy metabolism, oxidative metabolism, mitochondrial biogenesis, and function has been proven, as have changes in muscle fiber types [42]. Moreover, the PPARGC1A rs8192678 Gly/Gly genotype has been associated with more significant increases in anaerobic threshold [54], more slow muscle fibers [55], more mitochondria activity, and a greater $\mathrm{VO}_{2}$ peak after aerobic training than the PPARGC1A rs 8192678 Ser allele genotype. Another aspect is that plasmids bearing Gly or Ser at position 482 in the PGC- $1 \alpha$ protein showed that the PPARGC1A 482Ser variant was less efficient as a coactivator of the myocyte enhancer factor $2 \mathrm{C}$ (MEF2C), which is a transcription factor regulating glucose transportation in skeletal muscle [58]. The described structure of the PPARs and their coactivators, therefore, targets many aspects necessary for elite athletic performance and might be used for training method selection 
or nutritional strategies [4]. Our results in the association of PPARGC1A Gly428Ser rs8192678 with endurance elite status seemed to be controversial with respect to previous findings [22], resulting in that this genotype was somewhat related to the power oriented athletes. This difference might be due to the contradictory finding in the original studies and that previous meta-analyses did not separate the comparisons between elite and subelite vs. comparisons between elite and control groups.

\section{Materials and Methods}

\subsection{Review Process}

The review was performed according to the Preferred Reporting Items for Systematic Reviews and Meta-Analyses (PRISMA) [59] guidelines using the review protocol assigned in PROSPERO under Database No. CRD42018082236. The final article eligibility was assessed using the adapted "Strengthening the Reporting of Observational Studies in Epidemiology" (STROBE) checklist [60] (Table S1).

\subsection{Literature Search}

To find articles related to the role of PPAR polymorphisms in elite sports, we conducted a systematic computerized literature search on 20 August 2019, in PubMed (1940 to search date), Scopus (1823 to search date), and the Web of Science (1974 to search date). A combination of the following search terms was used: (PPAR) OR (peroxisome AND proliferator AND activated AND receptor) AND (sports) OR (physical AND activity) OR (endurance) OR (exercise) OR (performance) OR (movement). The search did not include comments, proceedings, editorial letters, conference abstracts, nor dissertations. Reviews were included for a manual search of their reference lists. A manual search of the reference lists of included articles was also performed (Figure 1).

\subsection{Literature Selection}

After identifying potential articles, the titles and abstracts were reviewed by two independent reviewers (P.S., M.P.) to select relevant articles for full-text screening according to the following inclusion criteria:

- Genotyping in PPARA, PPARG, PPARD, PPARGC1A, PPARGC1B, and genes.

- The population of athletes.

- Cross-sectional, cohort, case-control, intervention, control trial, or GWAS.

When the inclusion of articles was questionable, the reviewers agreed after a discussion. The full-text analyses of the relevant articles were performed by three independent reviewers (P.S., M.P., A.M.-S.) who also completed the data extraction form (Table S2). During the full-text screening, the following exclusion criteria were used:

(1) the full text was not available in English;

(2) the study did not contain an appropriate description of athlete performance status;

(3) the study did not include a specification of the selected sports discipline;

(4) the study did not report PPAR frequencies for elite athletes;

(5) the study was not reproducible by the methodological quality criteria.

\subsection{Qualitative Synthesis}

The result of the qualitative synthesis was based on the comparison of the type of participants in the original studies, where the highest importance was considered for comparison of elite athletes to subelite athletes. Then, the comparison between elite athletes and controls was considered as a supportive level of meaningful. Elite sports status was determined during full-text screening, where we used the original status definition of the author if it was under elite status determination [61]. 
The synthesis summarized three categories of sports disciplines by the dominant metabolic demand for the disciplines: strength and power oriented athletes, endurance oriented athletes, and mixed type of activity according to previous definitions $[25,30,46]$, where team sports such as soccer or ice-hockey were considered as mixed strength-power and endurance disciplines.

\section{Conclusions}

PPARs could be used for estimating the potential to achieve elite status in human physical performance in strength, power, team, and aerobic sports disciplines. Carrying specific PPARs alleles could provide a partial benefit for achieving elite sports status, but did not preclude achieving elite status if they were absent. The Ala allele in PPARG rs1801282 supported the achievement of elite athlete status in strength and power disciplines. The $\mathrm{C}$ allele in PPARA rs4253778 supported the achievement of elite athlete status in mixed strength and endurance soccer, and the G allele PPARA rs4253778 supported achievement in endurance athletes. The Gly allele in PPARGC1A rs8192678 and the C allele PPARD rs2016520 supported the achievement of elite athlete status in endurance sports disciplines.

Supplementary Materials: Supplementary materials can be found at http://www.mdpi.com/1422-0067/21/1/162/ s1 link.

Author Contributions: P.S., M.P. and A.Z. conceived of and designed the review; P.S., M.P. and A.M.-S. performed the review; P.S., M.P. and A.M.-S. analyzed the data; P.S., M.P., A.M.-S. and J.C. wrote the paper. All authors have read and agreed to the published version of the manuscript.

Funding: This research was funded by Charles University, grant number UNCE/HUM/03 and The Grant Agency of Czech Republic no GA19-12150S.

Acknowledgments: This study was supported by the UNCE/HUM/032, GACR GA19-12150S grant at Charles University.

Conflicts of Interest: The authors declare no conflict of interest.

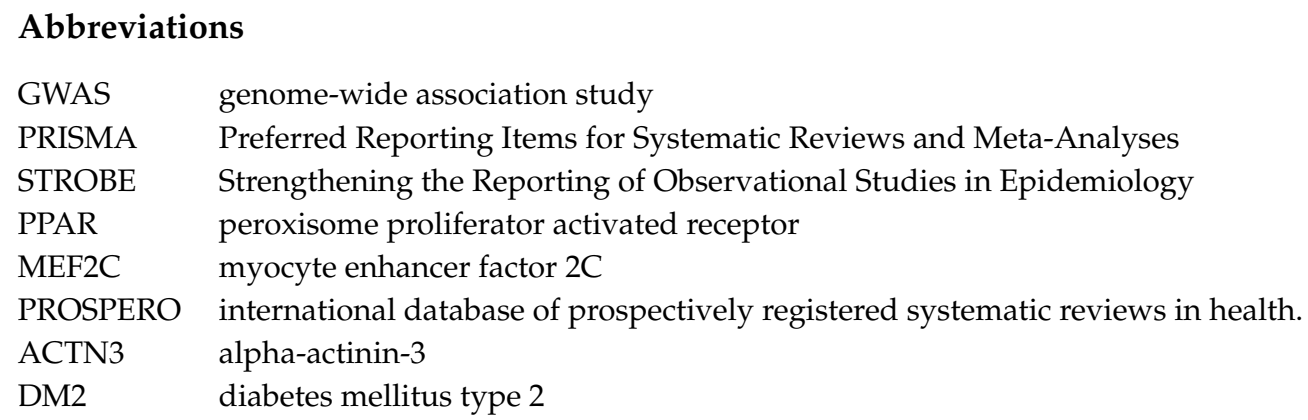

\section{References}

1. Semenova, E.A.; Fuku, N.; Ahmetov, I.I. Genetic profile of elite endurance athletes. In Sports, Exercise, and Nutritional Genomics; Elsevier Academic Press: London, UK, 2019; pp. 73-104.

2. Maciejewska-Skrendo, A.; Sawczuk, M.; Cięszczyk, P.; Ahmetov, I.I. Genes and power athlete status. In Sports, Exercise, and Nutritional Genomics; Elsevier Academic Press: London, UK, 2019; pp. 41-72.

3. Maciejewska-Skrendo, A.; Cięszczyk, P.; Chycki, J.; Sawczuk, M.; Smółka, W. Genetic Markers Associated with Power Athlete Status. J. Hum. Kinet. 2019, 68, 17. [CrossRef] [PubMed]

4. Petr, M.; Stastny, P.; Zajac, A.; Tufano, J.J.; Maciejewska-Skrendo, A. The Role of Peroxisome Proliferator-Activated Receptors and Their Transcriptional Coactivators Gene Variations in Human Trainability: A Systematic Review. Int. J. Mol. Sci. 2018, 19, 1472. [CrossRef] [PubMed]

5. Valeeva, E.V.; Ahmetov, I.I.; Rees, T. Psychogenetics and sports. Sports, Exercise, and Nutritional Genomics; Elsevier: Amsterdam, The Netherlands, 2019; pp. 147-165.

6. Kersten, S.; Desvergne, B.; Wahli, W. Roles of PPARs in health and disease. Nature 2000, 405, 421-424. [CrossRef] [PubMed] 
7. Pozzi, A.; Ibanez, M.R.; Gatica, A.E.; Yang, S.; Wei, S.; Mei, S.; Falck, J.R.; Capdevila, J.H. Peroxisomal proliferator-activated receptor- $\alpha$-dependent inhibition of endothelial cell proliferation and tumorigenesis. J. Biol. Chem. 2007, 282, 17685-17695. [CrossRef]

8. Dubuquoy, L.; Dharancy, S.; Nutten, S.; Pettersson, S.; Auwerx, J.; Desreumaux, P. Role of peroxisome proliferator-activated receptor $\gamma$ and retinoid $\mathrm{X}$ receptor heterodimer in hepatogastroenterological diseases. Lancet 2002, 360, 1410-1418. [CrossRef]

9. Cabrero, A.; Laguna, J.; Vazquez, M. Peroxisome proliferator-activated receptors and the control of inflammation. Curr. Drug Targets-Inflamm. Allergy 2002, 1, 243-248. [CrossRef]

10. Leonardini, A.; Laviola, L.; Perrini, S.; Natalicchio, A.; Giorgino, F. Cross-talk between PPAR and insulin signaling and modulation of insulin sensitivity. PPAR Res. 2009. [CrossRef]

11. Yessoufou, A.; Wahli, W. Multifaceted roles of peroxisome proliferator-activated receptors (PPARs) at the cellular and whole organism levels. Swiss Med Wkly. 2010, 140. [CrossRef]

12. Kliewer, S.; Forman, B.; Blumberg, B.; Ong, E.; Borgmeyer, U.; Mangelsdorf, D.; Umesono, K.; Evans, R.M. Differential expression and activation of a family of murine peroxisome proliferator-activated receptors. Proc. Natl. Acad. Sci. USA 1994, 91, 7355-7359. [CrossRef]

13. Manickam, R.; Wahli, W. Roles of peroxisome proliferator-activated receptor $\beta / \delta$ in skeletal muscle physiology. Biochimie 2017, 136, 42-48. [CrossRef]

14. Cagnin, S.; Chemello, F.; Ahmetov, I.I. Genes and response to aerobic training. In Sports, Exercise, and Nutritional Genomics; Elsevier Academic Press: London, UK, 2019; pp. 169-188.

15. Gleyzer, N.; Scarpulla, R.C. PGC-1-related coactivator (PRC), a sensor of metabolic stress, orchestrates a redox-sensitive program of inflammatory gene expression. J. Biol. Chem. 2011, 286, 39715-39725. [CrossRef] [PubMed]

16. Handschin, C.; Spiegelman, B.M. Peroxisome proliferator-activated receptor $\gamma$ coactivator 1 coactivators, energy homeostasis, and metabolism. Endocr. Rev. 2006, 27, 728-735. [CrossRef] [PubMed]

17. Franks, P.W.; Christophi, C.A.; Jablonski, K.A.; Billings, L.K.; Delahanty, L.M.; Horton, E.S.; Knowler, W.C.; Florez, J.C.; Diabetes Prevention Program Research Group. Common variation at PPARGC1A/B and change in body composition and metabolic traits following preventive interventions: The Diabetes Prevention Program. Diabetologia 2014, 57, 485-490. [CrossRef] [PubMed]

18. Ahmetov, I.I.; Williams, A.G.; Popov, D.V.; Lyubaeva, E.V.; Hakimullina, A.M.; Fedotovskaya, O.N.; Mozhayskaya, I.A.; Vinogradova, O.L.; Astratenkova, I.V.; Montgomery, H.E.; et al. The combined impact of metabolic gene polymorphisms on elite endurance athlete status and related phenotypes. Hum. Gen. 2009, 126, 751-761. [CrossRef] [PubMed]

19. Ahmetov, I.I.; Popov, D.V.; Mozhaiskaia, I.A.; Missina, S.S.; Astratenkova, I.V.; Vinogradova, O.L.; Rogozkin, V.A. Association of regulatory genes polymorphisms with aerobic and anaerobic performance of athletes. Rossǐ̌skii fiziologicheskǐ̌ zhurnal imeni IM Sechenova/Rossǐ̌skaia akademiia nauk 2007, 93, 837-843.

20. Franks, P.W.; Barroso, I.; Luan, J.; Ekelund, U.; Crowley, V.E.F.; Brage, S.; Sandhu, M.S.; Jakes, R.W.; Middelberg, R.P.; Harding, A.H.; et al. PGC-1 $\alpha$ Genotype Modifies the Association of Volitional Energy Expenditure with VंO2max. Med. Sci. Sports. Exerc. 2003, 35, 1998-2004. [CrossRef]

21. Petr, M.; Št'Astný, P.; Pecha, O.; Šteffl, M.; Šeda, O.; Kohlíková, E. PPARA intron polymorphism associated with power performance in 30-s anaerobic wingate test. PLoS ONE 2014, 9, e107171. [CrossRef]

22. Tharabenjasin, P.; Pabalan, N.; Jarjanazi, H. Association of PPARGC1A Gly428Ser (rs8192678) polymorphism with potential for athletic ability and sports performance: A meta-analysis. PLoS ONE 2019, 14, e0200967. [CrossRef]

23. Mathai, A.S.; Bonen, A.; Benton, C.R.; Robinson, D.L.; Graham, T.E. Rapid exercise-induced changes in PGC-1 $\alpha$ mRNA and protein in human skeletal muscle. J. Appl. Physiol. 2008, 105, 1098-1105. [CrossRef]

24. Nezhad, F.Y.; Verbrugge, S.A.J.; Schönfelder, M.; Becker, L.; De Angelis, M.H.; Wackerhage, H. Genes whose gain or loss-of-function increases endurance performance in Mice: A systematic literature review. Front. Physiol. 2019, 10, 262. [CrossRef]

25. Ahmetov, I.I.; Mozhayskaya, I.A.; Flavell, D.M.; Astratenkova, I.V.; Komkova, A.I.; Lyubaeva, E.V.; Tarakin, P.P.; Shenkman, B.S.; Vdovina, A.B.; Netreba, A.I.; et al. PPAR $\alpha$ gene variation and physical performance in Russian athletes. Eur. J. Appl. Physiol. 2006, 97, 103-108. [CrossRef] [PubMed]

26. Ahmetov, I.I.; Astratenkova, I.V.; Rogozkin, V.A. Association of a PPARD polymorphism with human physical performance. Mol. Biol. 2007, 41, 776-780. [CrossRef] 
27. Ahmetov, I.I.; Mozhayskaya, I.A.; Lyubaeva, E.V.; Vinogradova, O.L.; Rogozkin, V.A. PPARG Gene polymorphism and locomotor activity in humans. Bull. Exp. Biol. Med. 2008, 146, 630-632. [CrossRef] [PubMed]

28. Cieszczyk, P.; Sawczuk, M.; Maciejewska, A.; Ficek, K.; Eider, A. Variation in peroxisome proliferator activated receptor $\alpha$ gene in elite combat athletes. Eur. J. Sport. Sci. 2011, 11, 119-123. [CrossRef]

29. Cocci, P.; Pistolesi, L.; Guercioni, M.; Belli, L.; Carli, D.; Palermo, F.A. Genetic Variants and Mixed Sport Disciplines: A Comparison among Soccer, Combat and Motorcycle Athletes. Ann. Appl. Sport. Sci. 2019, 7, 1-9. [CrossRef]

30. Drozdovska, S.B.; Dosenko, V.E.; Ahmetov, I.I.; Ilyin, V.N. The association of gene polymorphisms with athlete status in Ukrainians. Biol. Sport 2013, 30, 163-167. [CrossRef]

31. Eynon, N.; Ruiz, J.R.; Meckel, Y.; Morán, M.; Lucia, A. Mitochondrial biogenesis related endurance genotype score and sports performance in athletes. Mitochondrion 2011, 11, 64-69. [CrossRef]

32. Eynon, N.; Meckel, Y.; Sagiv, M.; Yamin, C.; Amir, R.; Sagiv, M.; Goldhammer, E.; Duarte, J.A.; Oliveira, J. Do PPARGC1A and PPAR $\alpha$ polymorphisms influence sprint or endurance phenotypes? Scand. J. Med. Sci. Sports 2010, 20, e145-e150. [CrossRef]

33. Egorova, E.S.; Borisova, A.V.; Mustafina, L.J.; Arkhipova, A.A.; Gabbasov, R.T.; Druzhevskaya, A.M.; Astratenkova, I.V.; Ahmetov, I.I. The polygenic profile of Russian football players. J. Sports. Sci. 2014, 32, 1286-1293. [CrossRef]

34. Ginevičiene, V.; Pranckevičiene, E.; Milašius, K.; Kučinskas, V. Gene variants related to the power performance of the Lithuanian athletes. Cen. Eur. J. Biol. 2011, 6, 48-57. [CrossRef]

35. Eynon, N.; Alves, A.J.; Yamin, C.; Meckel, Y. PPARA intron 1 A/C polymorphism and elite athlete status. Eur. J. Sport Sci. 2011, 11, 177-181. [CrossRef]

36. Gineviciene, V.; Jakaitiene, A.; Tubelis, L.; Kucinskas, V. Variation in the ACE, PPARGC1A and PPARA genes in Lithuanian football players. Eur. J. Sport Sci. 2014, 14, S289-S295. [CrossRef] [PubMed]

37. Gineviciene, V.; Jakaitiene, A.; Aksenov, M.O.; Aksenova, A.V.; Druzhevskaya, A.M.; Astratenkova, I.V.; Egorova, E.S.; Gabdrakhmanova, L.J.; Tubelis, L.; Kucinskas, V.; et al. Association analysis of ACE, ACTN3 and PPARGC1A gene polymorphisms in two cohorts of European strength and power athletes. Biol. Sport 2016, 33, 199-206. [CrossRef] [PubMed]

38. Gonzalez-Freire, M.; Santiago, C.; Verde, Z.; Lao, J.I.; Olivan, J.; Gallego, F.G.; Lucia, A. Unique among unique. Is it genetically determined? Br. J. Sports Med. 2009, 43, 307-309. [CrossRef] [PubMed]

39. Grealy, R.; Herruer, J.; Smith, C.L.E.; Hiller, D.; Haseler, L.J.; Griffiths, L.R. Evaluation of a 7-gene genetic profile for athletic endurance phenotype in ironman championship triathletes. PLoS ONE 2015, 10, e0145171. [CrossRef] [PubMed]

40. Lucia, A.; Gomez-Gallego, F.; Barroso, I.; Rabadan, M.; Bandres, F.; San Juan, A.F.; Chicharro, J.L.; Ekelund, U.; Brage, S.; Earnest, C.P.; et al. PPARGC1A genotype (Gly482Ser) predicts exceptional endurance capacity in European men. J. Appl. Physiol. (1985) 2005, 99, 344-348. [CrossRef]

41. Maciejewska, A.; Sawczuk, M.; Cieszczyk, P. Variation in the PPARalpha gene in Polish rowers. J. Sci. Med. Sport 2011, 14, 58-64. [CrossRef]

42. Maciejewska, A.; Sawczuk, M.; Cieszczyk, P.; Mozhayskaya, I.A.; Ahmetov, I.I. The PPARGC1A gene Gly482Ser in Polish and Russian athletes. J. Sports Sci. 2012, 30, 101-113. [CrossRef]

43. Maciejewska-Karlowska, A.; Sawczuk, M.; Cieszczyk, P.; Zarebska, A.; Sawczyn, S. Association between the Pro12Ala Polymorphism of the Peroxisome Proliferator-Activated Receptor Gamma Gene and Strength Athlete Status. PLoS ONE 2013, 8, e67172. [CrossRef]

44. Maciejewska-Karlowska, A.; Hanson, E.D.; Sawczuk, M.; Cieszczyk, P.; Eynon, N. Genomic haplotype within the Peroxisome Proliferator-Activated Receptor Delta (PPARD) gene is associated with elite athletic status. Scand. J. Med. Sci. Sports 2014, 24, e148-e155. [CrossRef]

45. Muniesa, C.A.; González-Freire, M.; Santiago, C.; Lao, J.I.; Buxens, A.; Rubio, J.C.; Martín, M.A.; Arenas, J.; Gomez-Gallego, F.; Lucia, A. World-class performance in lightweight rowing: Is it genetically influenced? A comparison with cyclists, runners and non-athletes. Br. J. Sports Med. 2010, 44, 898-901. [CrossRef] [PubMed]

46. Peplonska, B.; Adamczyk, J.G.; Siewierski, M.; Safranow, K.; Maruszak, A.; Sozanski, H.; Gajewski, A.K.; Zekanowski, C. Genetic variants associated with physical and mental characteristics of the elite athletes in the Polish population. Scand. J. Med. Sci. Sports 2017, 27, 788-800. [CrossRef] [PubMed] 
47. Santiago, C.; Ruiz, J.R.; Muniesa, C.A.; González-Freire, M.; Gómez-Gallego, F.; Lucia, A. Does the polygenic profile determine the potential for becoming a world-class athlete? Insights from the sports of rowing. Scand. J. Med. Sci. Sports 2010, 20, e188-e194. [CrossRef] [PubMed]

48. Tsianos, G.I.; Evangelou, E.; Boot, A.; Carola Zillikens, M.; Van Meurs, J.B.J.; Uitterlinden, A.G.; Ioannidis, J.P. Associations of polymorphisms of eight muscle-Or metabolism-related genes with performance in Mount Olympus marathon runners. J. Appl. Physiol. 2010, 108, 567-574. [CrossRef] [PubMed]

49. Tural, E.; Kara, N.; Agaoglu, S.A.; Elbistan, M.; Tasmektepligil, M.Y.; Imamoglu, O. PPAR- $\alpha$ and PPARGC1A gene variants have strong effects on aerobic performance of Turkish elite endurance athletes. Mol. Biol. Rep. 2014, 41, 5799-5804. [CrossRef] [PubMed]

50. Yvert, T.; Miyamoto-Mikami, E.; Murakami, H.; Miyachi, M.; Kawahara, T.; Fuku, N. Lack of replication of associations between multiple genetic polymorphisms and endurance athlete status in Japanese population. Physiol. Rep. 2016, 4, e13003. [CrossRef]

51. Eynon, N.; Birk, R.; Meckel, Y.; Lucia, A.; Nemet, D.; Eliakim, A. Physiological variables and mitochondrial-related genotypes of an athlete who excels in both short and long-distance running. Mitochondrion 2011, 11, 774-777. [CrossRef]

52. Lucia, A.; Oliván, J.; Gómez-Gallego, F.; Santiago, C.; Montil, M.; Foster, C. Citius and longius (faster and longer) with no $\alpha$-actinin-3 in skeletal muscles? Br. J. Sports Med. 2007, 41, 616-617. [CrossRef]

53. Aksenov, M.O.; Ilyin, A.B. Training process design in weightlifting sports customized to genetic predispositions. Teoriya i Praktika Fizicheskoy Kultury 2017, 6, 75-77.

54. Stefan, N.; Thamer, C.; Staiger, H.; Machicao, F.; Machann, J.; Schick, F.; Venter, C.; Niess, A.; Laakso, M.; Fritsche, A.; et al. Genetic variations in PPARD and PPARGC1A determine mitochondrial function and change in aerobic physical fitness and insulin sensitivity during lifestyle intervention. J. Clin. Endocrin. Metabol. 2007, 92, 1827-1833. [CrossRef]

55. Steinbacher, P.; Feichtinger, R.G.; Kedenko, L.; Kedenko, I.; Reinhardt, S.; Schönauer, A.L.; Leitner, I.; Sänger, A.M.; Stoiber, W.; Kofler, B.; et al. The single nucleotide polymorphism Gly482Ser in the PGC-1 $\alpha$ gene impairs exercise-induced slow-twitch muscle fibre transformation in humans. PLoS ONE 2015, 10, e0123881. [CrossRef] [PubMed]

56. Ring-Dimitriou, S.; Kedenko, L.; Kedenko, I.; Feichtinger, R.G.; Steinbacher, P.; Stoiber, W.; Forster, H.; Felder, T.K.; Muller, E.; Kolfer, B. Does genetic variation in PPARGC1A affect exercise-induced changes in ventilatory thresholds and metabolic syndrome? J. Exerc. Physiol. Online 2014, 17, 1-18.

57. Hautala, A.J.; Leon, A.S.; Skinner, J.S.; Rao, D.C.; Bouchard, C.; Rankinen, T. Peroxisome proliferator-activated receptor-delta polymorphisms are associated with physical performance and plasma lipids: The HERITAGE Family Study. Am. J. Physiol. Heart Circ. Physiol. 2007, 292, H2498-H2505. [CrossRef] [PubMed]

58. Zhang, S.-L.; Lu, W.-S.; Yan, L.; Wu, M.-C.; Xu, M.-T.; Chen, L.-H.; Cheng, H. Association between peroxisome proliferator-activated receptor-gamma coactivator-1alpha gene polymorphisms and type 2 diabetes in southern Chinese population: Role of altered interaction with myocyte enhancer factor 2C. Chin. Med. J. 2007, 120, 1878-1885. [CrossRef]

59. Moher, D.; Schulz, K.F.; Simera, I.; Altman, D.G. Guidance for developers of health research reporting guidelines. PLoS Med. 2010, 7, e1000217. [CrossRef]

60. Von Elm, E.; Altman, D.G.; Egger, M.; Pocock, S.J.; Gøtzsche, P.C.; Vandenbroucke, J.P. The Strengthening the Reporting of Observational Studies in Epidemiology (STROBE) Statement: Guidelines for reporting observational studies. Prev. Med. 2007, 45, 247-251. [CrossRef]

61. Swann, C.; Moran, A.; Piggott, D. Defining elite athletes: Issues in the study of expert performance in sports psychology. Psych. Sport Exerc. 2015, 16, 3-14. [CrossRef]

(C) 2019 by the authors. Licensee MDPI, Basel, Switzerland. This article is an open access article distributed under the terms and conditions of the Creative Commons Attribution (CC BY) license (http://creativecommons.org/licenses/by/4.0/). 